\title{
Implementation of a structured information transfer checklist improves postoperative data transfer after congenital cardiac surgery
}

\author{
A. Karakaya1 , A. Moerman'1, H. Peperstraete ${ }^{2}$, P. Wouters ${ }^{1}$, S. De Hert ${ }^{1}$
}

Department of Anaesthesiology ${ }^{1}$, Department of Cardiac Intensive Care Unit ${ }^{2}$, Ghent University Hospital, Ghent, Belgium

\section{Context}

With patient transfer from one unit to another, it is of prime importance to convey a complete picture of the patient's situation to minimize the risk of medical errors and to provide optimal patient care

\section{Objective}

To test the hypothesis that implementation of a standardised checklist used during verbal patient handover could improve postoperative data transfer after congenital cardiac surgery

\section{Design}

- Prospective, pre-/postinterventional clinical study

- Approval ethical committee

- Forty-eight patients younger than 16 years undergoing heart surgery

\section{Interventions}

A standardised CHECKLIST was developed containing all data that according to the investigators should be communicated during handover of a paediatric cardiac surgery patient from the operating room to the intensive care unit

Main outcome
measures

- Data transfer during the postoperative handover BEFORE and AFTER implementation of the checklist

- Duration of handover, number of interruptions, irrelevant data and confusing data

- Assessment of the handover by using VAS

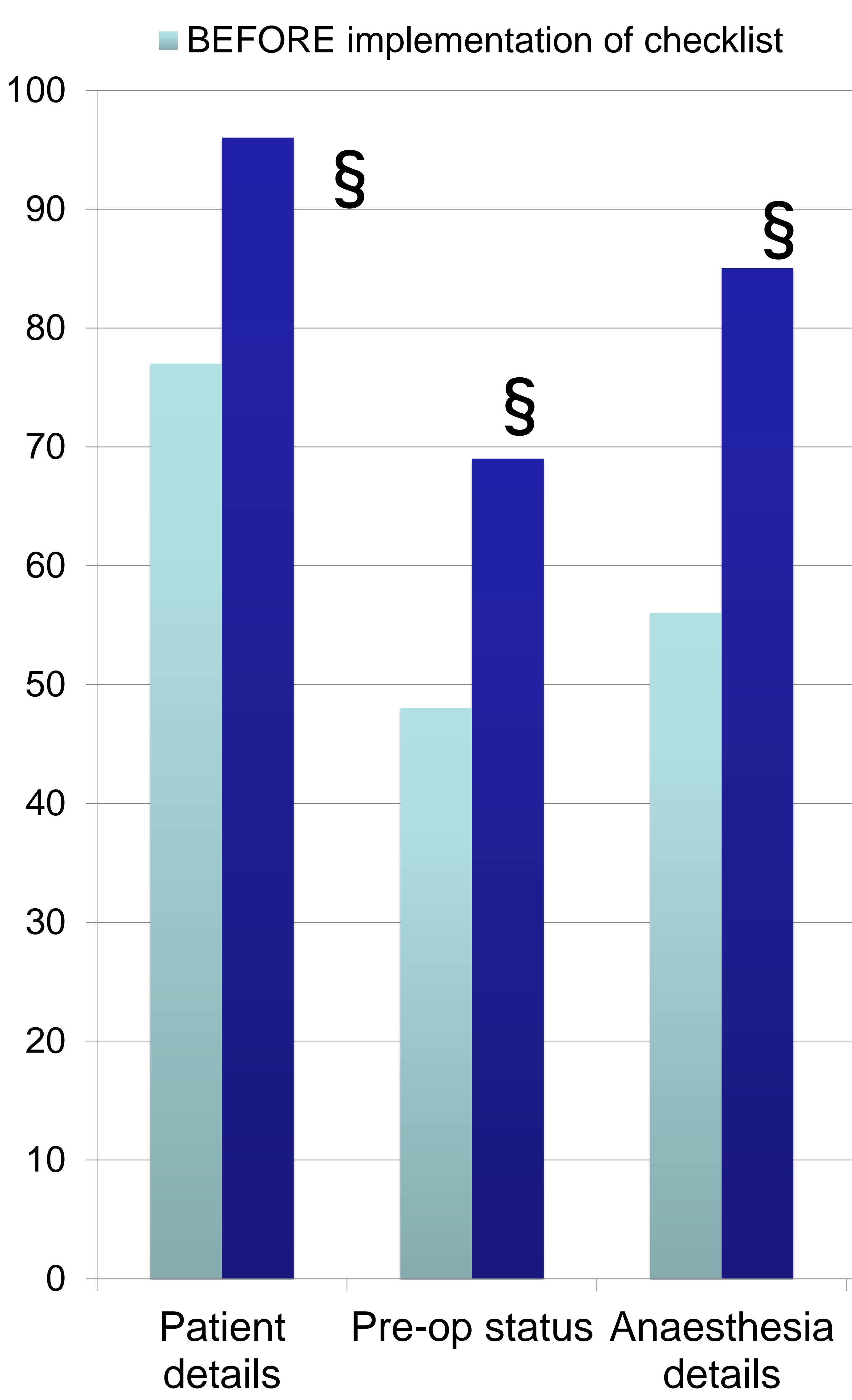

\section{CONCLUSION}

\section{Results}

- After implementation of the information transfer checklist, the overall data transfer increased from $48 \%$ to $73 \%(p<0.001)$

- The duration of data transfer decreased from a median of $6 \mathrm{~min}$ (range 2-16 $\mathrm{min}$ ) to $4 \mathrm{~min}$ (range $2-19 \mathrm{~min})(p=0.04)$

- The overall handover assessment by the intensive care nursing staff improved significantly $(p=0.004)$

- AFTER implementation of checklist
$\S p \leq 0.001$

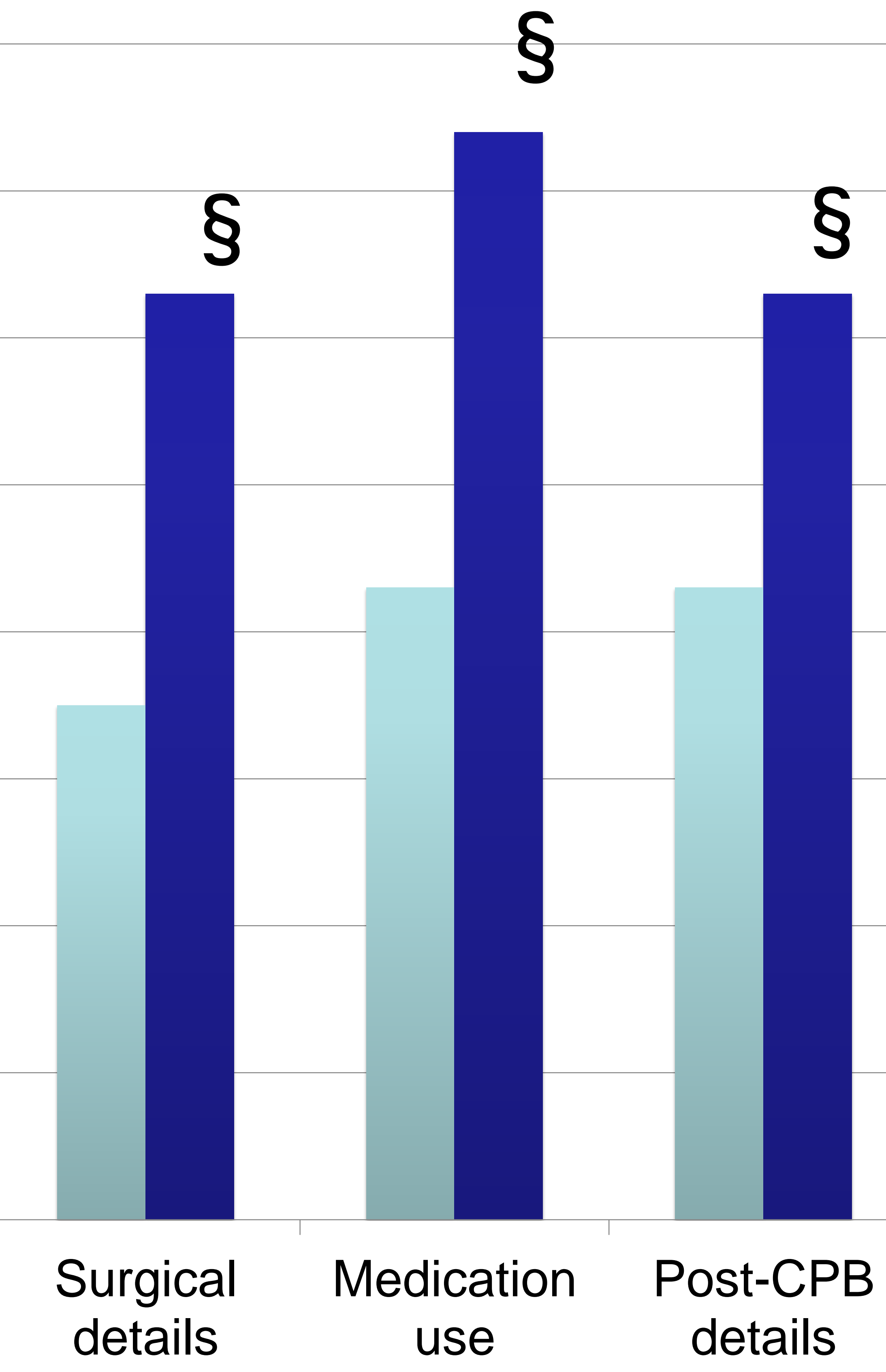

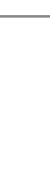
tails

\footnotetext{
Implementation of an information transfer

checklist in postoperative paediatric cardiac surgery

patients resulted in a more complete transfer of information with a decrease in the handover duration
}

\section{CONCLUSION}

